RBEn, "32 : 61-74, 1979

\title{
PARTICIPAÇÃO DA ENFERMAGEM NO PROGRAMA MATERNO-INFANTIL DA SECRETARIA DE SAÚdE DO PARÁ
}

Marisa Correia Hirata

\begin{tabular}{l|l|}
$/ 06$ \\
\hline
\end{tabular}

HIRATA, M.C. - Participação da enfermagem no programa materno-infantil da Secretaria de Saúde do Pará. Rev. Bras. Enf.; DF, 32 : 61-74, 1979.

\section{RESUMO}

Em linhas gerais, o Programa Materno-Infantil da SESPA, concentra a assistência prestada, principalmente nos niveis de Prevenção Primária, com a participação da Enfermagem em todas as atividades desenvolvidas.

Para operacionalizar o referido programa, foram organizados calendários de atendimento, fluxogramas e formulou-se metas considerando os recursos disponiveis.

O grupo Materno-Infantil vem sendo trabalhado numa abrangência crescente, envolvendo o periodo pré-natal, parto e puerpério, seguido de captação precoce e atendimento da criança menor de 1 ano, dando sequiência à criança de 1 a 4 anos e de 5 a 14 anos.

A observação de coeficientes vitais relativos aos últmos 3 (três) anos, levanos a supor que as atividades desenvolvidas estão contribuindo para a melho- ria do nivel de saúde da população materno-infantil do Pará.

\section{INTRODUÇAO}

O Programa Materno-Infantil cons-titui um dos programas prioritários do Plano Decenal de Saúde para as Américas, do Plano Nacional de Desenvolvimento do $\mathrm{Pais}$ e, conseqüentemente, entre os de prioridade do Estado.

$\mathrm{Na}$ assistência de saúde oferecida à população alvo do referido programa, estão em primeiro plano as de Prevenção Primária (Promoção da Saúde e Proteção Específica), onde a enfermagem tem uma atuação marcante, dando continuidade a um trabalho que, historicamente, remonta os primórdios da Saúde Pública, por ocasião da revolução industrial na Europa quando enfermeiras iniciaram trabalhos de atenção em domicílio às famílias de operários. 
HIRATA, M.C. - Participaçāo da enfermnagem no programa materno-infantil da Secretaria de Saúde do Pará. Rev. Bras. Enf.; DF, 32 : 61-74, 1979.

1. DIAGNÓSTICO DE SAÚdE

1.1 - População Materno-Infantil

O grupo Materno-Infantil apresenta, no nosso Estado, a seguinte dis. tribuiçāo percentual:

Tabela I

DISTRIBUIÇAO PERCENTUAL DA POPULAÇAO MATERNO-INFANTIL DO PARA - 1977

\begin{tabular}{|c|c|c|}
\hline $\begin{array}{l}\text { População total } \\
\text { Grupo M.I. }\end{array}$ & $\begin{array}{l}2.885 .540 \\
2.160 .346 \\
\end{array}$ & $\begin{array}{r}100,0 \text { \& } \\
74,8 \backsim \\
\end{array}$ \\
\hline 0 a 4 anos & 505.546 & 17,5 \% \\
\hline 5 a 14 " & 841.444 & 29,2 \% \\
\hline 15 a 19 " (fem) & 165.629 & 5,78 \\
\hline 15 a 49 " (fem) & 647.727 & 22,48 \\
\hline
\end{tabular}

Como vimos, $74,8 \%$ da população totaldo Pará.

FIG. 1

População Materno-Infantil

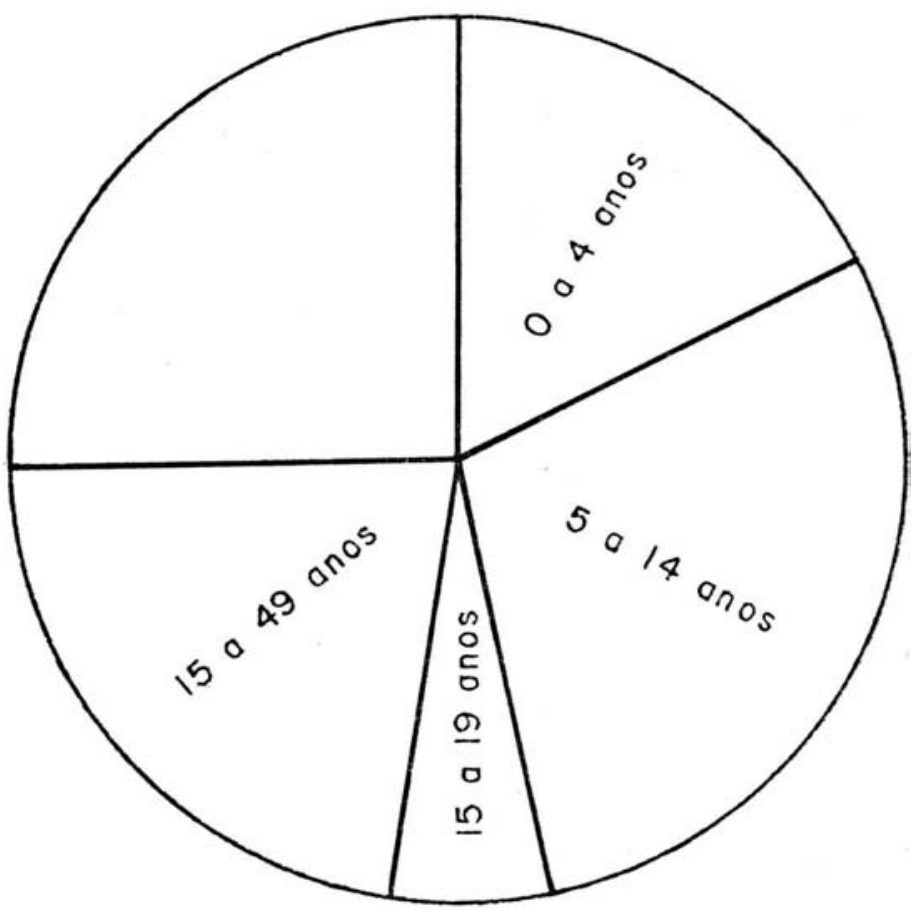


HIRATA, M.C. - Participaçāo da enfermagem no programa materno-infantil da Secretaria de Saúde do Pará. Rev. Bras. Enf.; DF, 32 : 61-74, 1979.

\section{2 - Nível de Saúde}

Nos últimos três anos, observou-se uma diminuição nos coeficientes de mortalidade materna $(1,8)$, não acontecendo o mesmo em relaçāo à mortalidade infantil $(68,0)$. Em levantamentos recentes o Setor de Informática desta Secretaria, verificou-se situação nos óbitos em menores de 1 ano:

Obituário em menores de 1 ano

Belém - 1977

FIG. 2

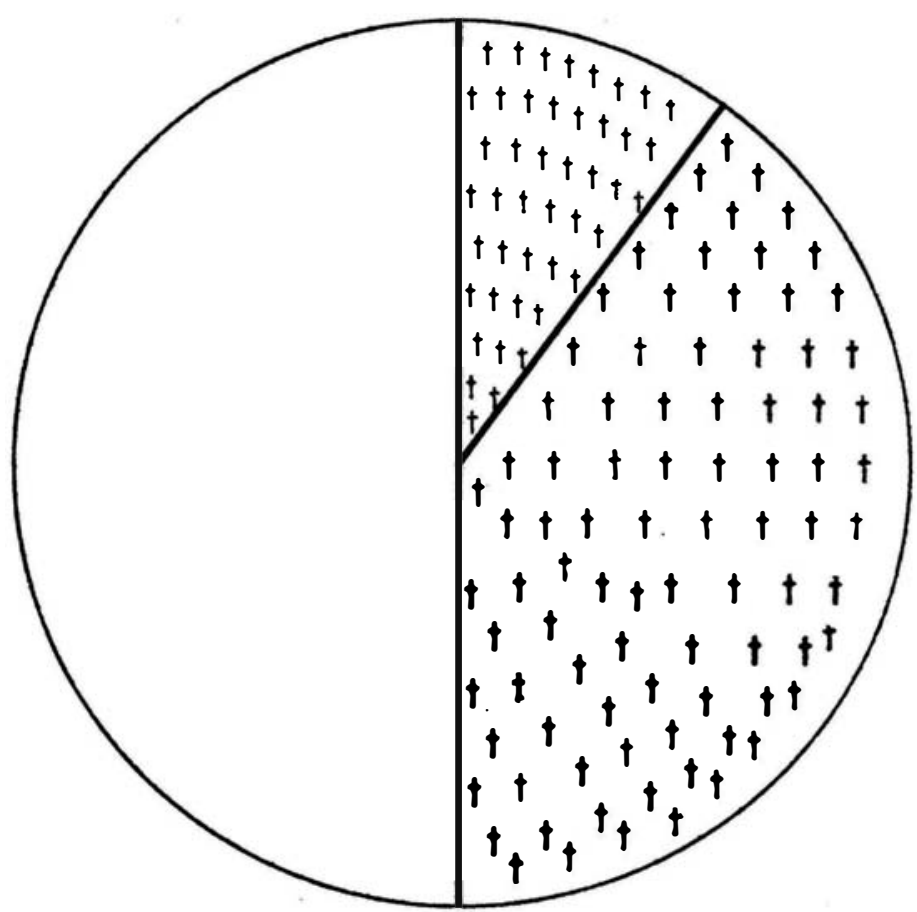

De cada 100 óbitos em menores de 1 ano, 50\% ocorre antes da criança completar 2 meses e 40\% antes de 1 mês de vida. 
HIRATA, M.C. - Participação da enfermagem no programa materno-infantil da Secretaria de Saúde do Pará. Rev. Bras. Enf.; DF, 32 : 61-74, 1979.

T A B E L A 2

Distribuição Percentual de óbitos de Menores de 1 ano

Até Novembro/77

\begin{tabular}{|c|c|c|}
\hline Faixa de Idade & No & \multicolumn{1}{|c|}{} \\
\hline-1 mês & 727 & 40,00 \\
1 mês & 182 & 10,00 \\
$2-11$ mêses & 906 & 50,00 \\
Total & 1.815 & 100,00 \\
\hline
\end{tabular}

1.3 - Recursos Médico-Sanitários

A Secretaria de Saúde possui 235 Unidades Sanitárias, sendo que $37,45 \%$ delas oferecem assistência materno-infantil com programação definida, avaliaçāo e controle. Congrega 20 instituiçōes produtoras de serviços ao grupo em pauta, quer particulares ou governamentais.

1.4 - Recursos Humanos (de enfermagem)

O quadro relativo ao pessoal de enfermagem da Secretaria (*) é o seguinte:

\begin{tabular}{|l|r|r|r|}
\hline & & \multicolumn{2}{|c|}{ P M I } \\
\cline { 3 - 4 } Categòria Profissional & Total & No & 8 \\
\hline Enfermeiro & 60 & 43 & 71,67 \\
Aux. de Enfermagem & 77 & 71 & 92,21 \\
Visitadora & 53 & 49 & 92,45 \\
Atendente & 708 & 622 & 87,85 \\
\hline
\end{tabular}

(*) até março de 1978

1.5 - Conclusão Diagnóstica

Numa rápida visão do problema, verificamos que a assistência materna apresenta coeficientes satisfatórios, porém na assistência infantil, os coeficientes de mortalidade estāo altos. Lembramos também que fatores extra-setoriais influenciam esta situação, tais como a renda per capita, educação, saneamento básico, infra-estruturas sociais, etc. 
HIRATA, M.C. - Participaçåo da enfermagem no programa materno-infantil da Secreta-: ria de Saúde do Pará. Rev. Bras. Enf.; DF, 32 : 61-74, 1979.

2. OBJETIVOS DO P.M.I.

Contribuir para a redução da morbi-mortalidade do grupo M.I.

Levar o grumo a uma par.
ticipação consciente è
efetiva na pramosão da
saúde.

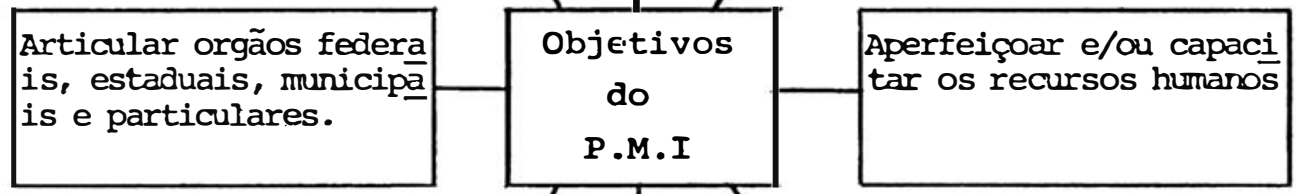

Ampliar e melhorar a $\propto \underline{0}$ bertura e os padrōes de produção das Unídades Sanitấrias.

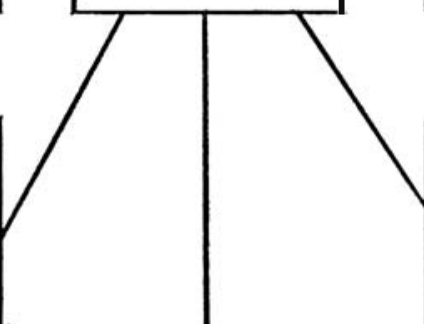

Efetivar processos sistemátios de planejamen to controle e avaliação

Contribuir para maior produtividade com o ingresso de novas e mais hígidas gerações na força de trabalho

\section{OPERACIONALIZAÇAO}

Para operacionalizar um programa do porte do P.M.I., usou-se a estratégia de atingir progressivamente a população alvo, atendendo-se prioridades do próprio grupo.

Para o ano em curso programou-se cobrir $72 \%$ dos municípios do Estado, envolvendo os seguintes grupos: 
HIRATA, M.C. - Participaçāo da enfermagem no programa materno-infantil da Secretaria de Saúde do Pará. Rev. Bras. Enf.; DF, 32 : 61-74, 1979.

\section{OPERALIZAÇÃO DO P.M.I.}

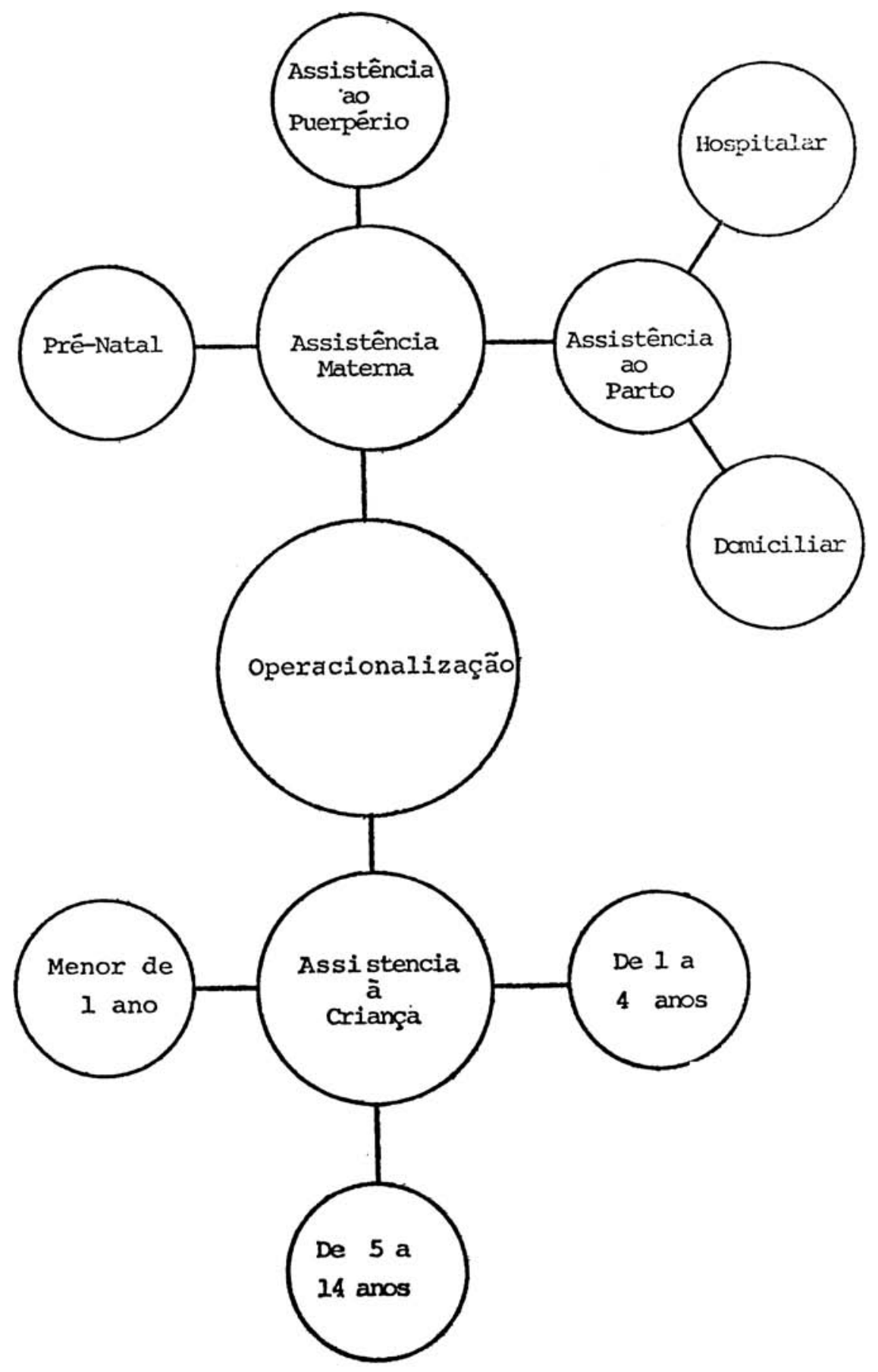


HIRATA, M.C. - Participaçāo da enfermagem no programa materno-infantil da Secretaria de Saúde do Pará. Rev. Bras. Enf.; DF, 32 : 61-74, 1979.

As açōes de saúde oferecidas ao grupo M.I. contam em $100 \%$ com a participação da enfermagem, nas tarefas fins.

\begin{tabular}{|c|c|c|c|c|c|c|c|c|c|c|c|}
\hline \multirow{2}{*}{$T A R E F A S$} & \multicolumn{8}{|c|}{$\begin{array}{c}\text { Equlpe } \\
\text { Multiprofissional }\end{array}$} & \multicolumn{3}{|c|}{$\begin{array}{l}\text { Grupo } \\
\text { M.I. }\end{array}$} \\
\hline & $E$ & $\mathrm{AE}$ & A & M & $\mathrm{O}$ & L & AS & ou & G & $\mathrm{P}$ & C \\
\hline \multirow{4}{*}{ 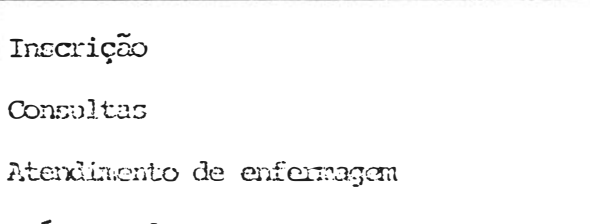 } & & & $\mathbf{x}$ & & & & & & $\mathbf{x}$ & & $x$ \\
\hline & $\mathbf{x}$ & & & $\mathbf{x}$ & $\mathbf{x}$ & & & & $\mathbf{x}$ & $x$ & $x$ \\
\hline & $\mathbf{x}$ & $\mathrm{x}$ & $\mathbf{x}$ & & & & & & $\mathbf{x}$ & & $\mathrm{x}$ \\
\hline & & & $\mathbf{x}$ & & & & & & $\mathbf{x}$ & & $x$ \\
\hline Fré-consulta & & & $\mathbf{x}$ & $\mathbf{x}$ & & & & & 25 & $\mathrm{x}$ & $\therefore$ \\
\hline E'ĆE-consulta & $x$ & $x$ & & & & & & & $\mathbf{x}$ & $x$ & $\mathrm{x}$ \\
\hline Visita demiciliar & & $x$ & $\mathbf{x}$ & & & & & & $\mathbf{x}$ & & $x$ \\
\hline Siplenkntagäo Ainnentar e rutricional & $\mathbf{x}$ & & & $\mathbf{x}$ & & & & & $\mathbf{x}$ & $x$ & $x$ \\
\hline \multirow{2}{*}{ Encwininaneritos ce Rotina } & $\mathbf{x}$ & $\mathbf{x}$ & $\mathrm{x}$ & $\mathbf{x}$ & & & & & $x$ & & $\dot{x}$ \\
\hline & & & & & & $\mathbf{x}$ & & & $\mathrm{x}$ & & $x$ \\
\hline Evarios Laboratoriais (urina - VDRL) & $\mathbf{x}$ & $\mathbf{x}$ & $\mathbf{x}$ & $\mathbf{x}$ & & & & & $x$ & $\mathrm{x}$ & \\
\hline Preventive do Cäncer & $\mathbf{x}$ & $\mathbf{x}$ & $\mathbf{x}$ & $\mathbf{x}$ & $\mathbf{x}$ & $\mathbf{x}$ & $\mathbf{x}$ & $\cdot x$ & $\mathbf{x}$ & $\mathbf{x}$ & $\mathbf{x}$ \\
\hline \multirow{2}{*}{$\begin{array}{l}\text { Educação para a saúcle } \\
\text { Orientacão de Parteiras Ieigas }\end{array}$} & $\mathbf{x}$ & $\mathbf{x}$ & & & & & & & & & \\
\hline & $\mathbf{x}$ & $\mathbf{x}$ & $\mathbf{x}$ & & & & & & & & \\
\hline
\end{tabular}

E - Enfermeira

$\mathrm{AE}$ - Auxiliar de Enfermagem

A - Atendente

M - Médico

O - Odontólogo

L - Laboratório

AS - Assistente Social

Ou - Outros

G - Gestante

P - Puerpera

C Criança. 
HURATA; M.C. - Participação da enfermagem no programa materno-infantil da Secretaria de Saúde do Pará. Rev. Bras. Enf.; DF, 32 : 61-74, 1979.

\section{ASSISTENCIA MATERNA}

$\mathrm{Na}$ assistência materna, temos:

4.1 - Pré-Natal que, no atendimento da gestante de baixo risco segue 0 seguinte calendário:

\begin{tabular}{|c|c|c|c|}
\hline $\begin{array}{c}\text { Idade da gestação } \\
\text { la. Consulta }\end{array}$ & \multicolumn{3}{|c|}{ Consultas subsequentes } \\
\hline Enf ${ }^{a}$ & Médico & $\operatorname{Enf}{ }^{a}$ & Médico \\
\hline 29 mês & 48 mês & 78 mês & 98 mês \\
\hline 30 mês & 58 mês & 78 mês & 98 mês \\
\hline 49 mês & 68 mês & 88 mês & 98 mês \\
\hline 58 mês & 78 mês & 88 mês & 98 mês \\
\hline 69 mês & 78 mês & 88 mês & 98 mês \\
\hline 78 mês & 88 mês & 98 mês * & \\
\hline 88 mês & 98 mês & - & \\
\hline 98 mês * & - & - & \\
\hline
\end{tabular}

* Fncaminhar ao médico

\section{2 - Assistência ao Parto}

4.2.1 - O parto hospitalar é realizado atualmente através de convênio com a Santa Casa de Misericórdia, numa enfermaria com o sistema "rooming-in".

4.2.2 - Assistência ao Parto Domiciliar - O parto domiciliar, está restrito quase que totalmente à área rural e, na maioria, efetuado através das "Parteiras Leigas", as quais recebem da enfermagem especial atenção, num trabalho onde foram treinadas nos três últimos anos 795 parteiras, recebendo todas elas bolsa equipada, ficando também sob controle da Unidade Sanitária.

Dentro de um conexto atual, a assistênncia oferecida é a seguinte: 
HIRATA, M.C. - Participaçāo da enfermagem no programa materno-infantil da Secretaria de Saúde do Pará. Rev. Bras. Enf.; DF, 32 : 61-74, 1979.

\section{FLUXOGRAMA \\ GESTANTE}

\section{TRIAGEM}

- 1

GRUPO DE ORTENTAÇÃO E INTERP.

SERVIÇO SOCIAL

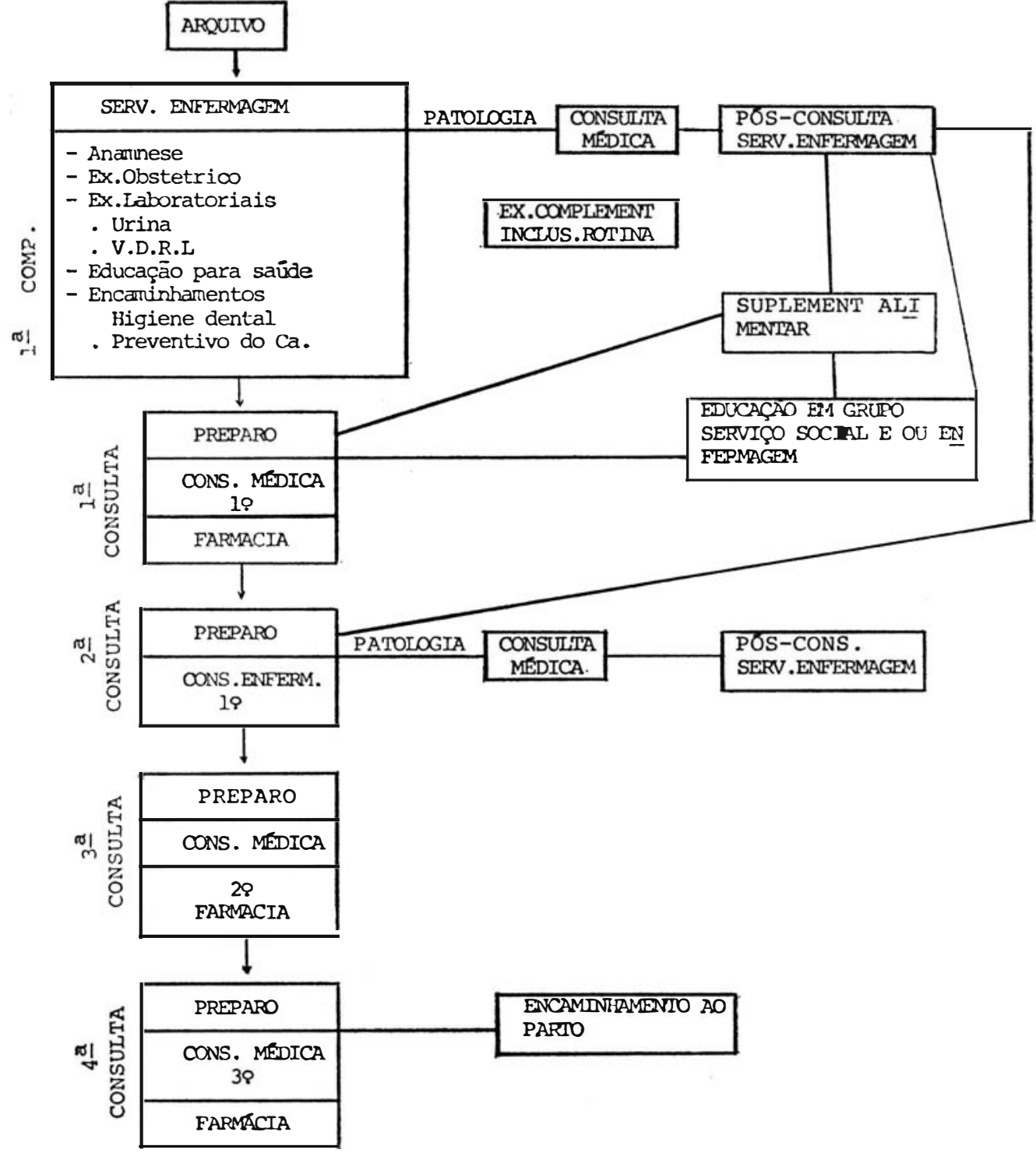


HIRATA, M.C. - Participaçāo da enfermagem no programa materno-infantil da secretaria de Saúde do Pará. Rev. Bras. Enf.; DF, 32 : 61-74, 1979.

\section{ASSISTENCIA AO PARTO DOMICILIAR}

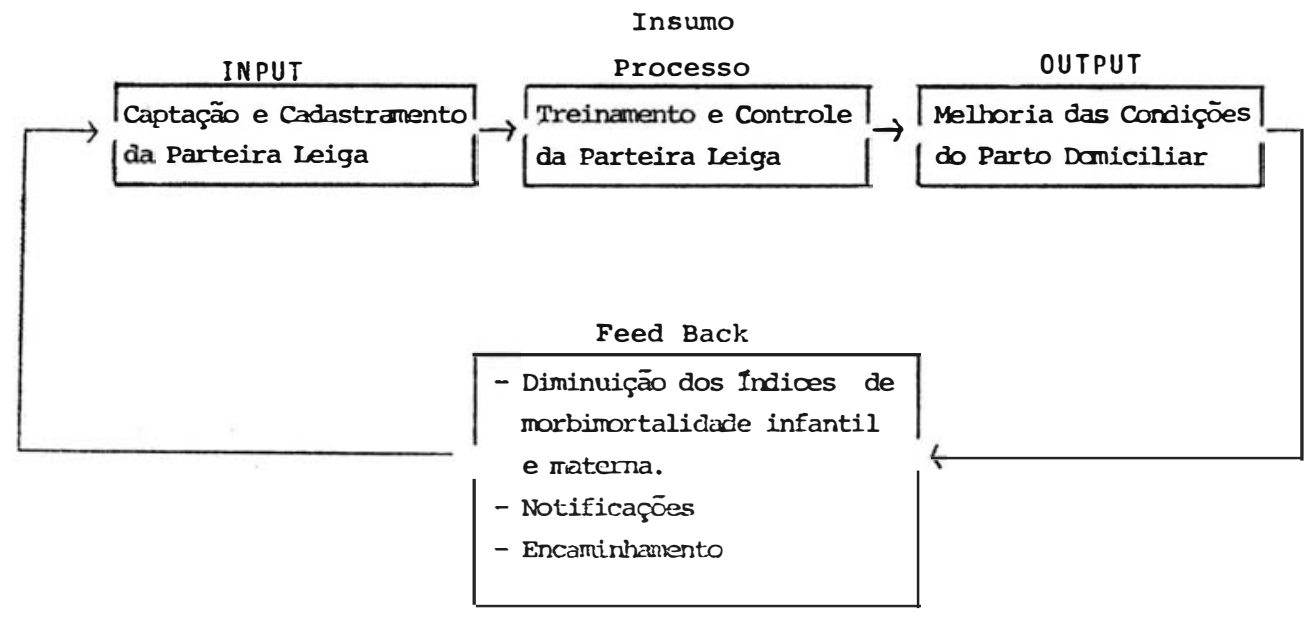

4.3 - Assistência ao Puerpério

As puérperas que realizam parto hospitalar, são orientadas pela enfermagem sobre os primeiros cuidados com o recém-nascido, incluindo higiene corporal, aleitamento materno, curativo umbilical, etc., recebendo inclusive material necessário para este fim.

Programou-se também uma visita domicilária no puerpério imediato ao binômio mãe-filho e uma consulta médica na Unidade Sanitária, ocasiāo em que será captado o recém-nascido para a puericultura 
HIRATA, M.C. - Participação da enfermagem no programa materno-infantil da Secretaria de Saúde do Pará. Rev. Bras. Ene.; DF, 32 : 61-74, 1979.

\section{ASSISTENCIA A CRIANÇA}

\section{1 - Criança de 0 a 1 ano}

Na assistência à criança de 0 a 1 ano, efetua-se a puericultura, conforme o seguinte calendário:

\section{CALENDARIO INFANTIL}

(0 a 1 ano)

\begin{tabular}{|c|c|c|c|c|c|}
\hline \multirow{2}{*}{ Ano } & \multirow{2}{*}{ Meses } & \multicolumn{3}{|c|}{$C O N$ T R O L E } & \multirow[b]{2}{*}{ Labor. } \\
\hline & & Médico & Enferm. & Imuniz. & \\
\hline \multirow{8}{*}{10} & 19 & & $\mathbf{X}$ & $\mathrm{X}$ & \\
\hline & 20 & $\mathrm{X}$ & & $\mathrm{X}$ & \\
\hline & 39 & & $\mathrm{X}$ & $\mathrm{X}$ & \\
\hline & 49 & $X$ & & $\mathrm{X}$ & \\
\hline & 69 & & $\mathrm{X}$ & $X$ & \\
\hline & 70 & & & $\mathrm{X}$ & \\
\hline & 89 & $\mathbf{X}$ & & $\mathrm{X}$ & \\
\hline & $12 \circ$ & & $x$ & & $\mathrm{X}$ \\
\hline
\end{tabular}


HIRATA, M.C. - Participação da enfermagem no programa materno-infantil da Secretaria de Saúde do Pará. Rev. Bras. Enf.; DF, 32:61-74, 1979.

FLUXOGRAMA

CRIANCCA MENOR DE 1 ANO

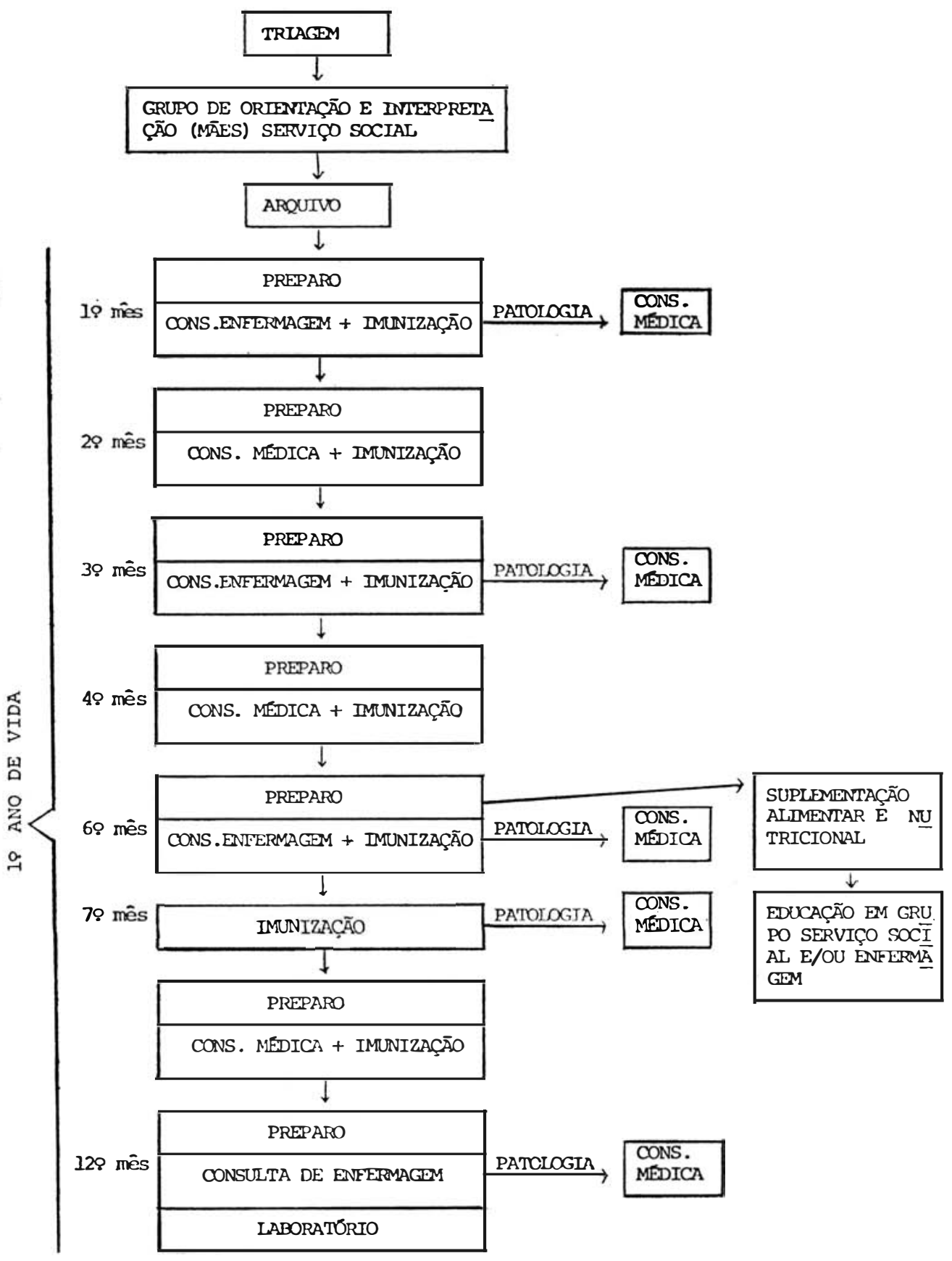


HIRATA, M.C. - Participaçāo da enfermagem no programa materno-infantil da Secretaria de Saúde do Pará. Rev. Bras. Enf.; DF, 32:61-74, 1979.

\section{FLUXOGRAMA}

CRIANCCA DE 1 A 4 ANOS

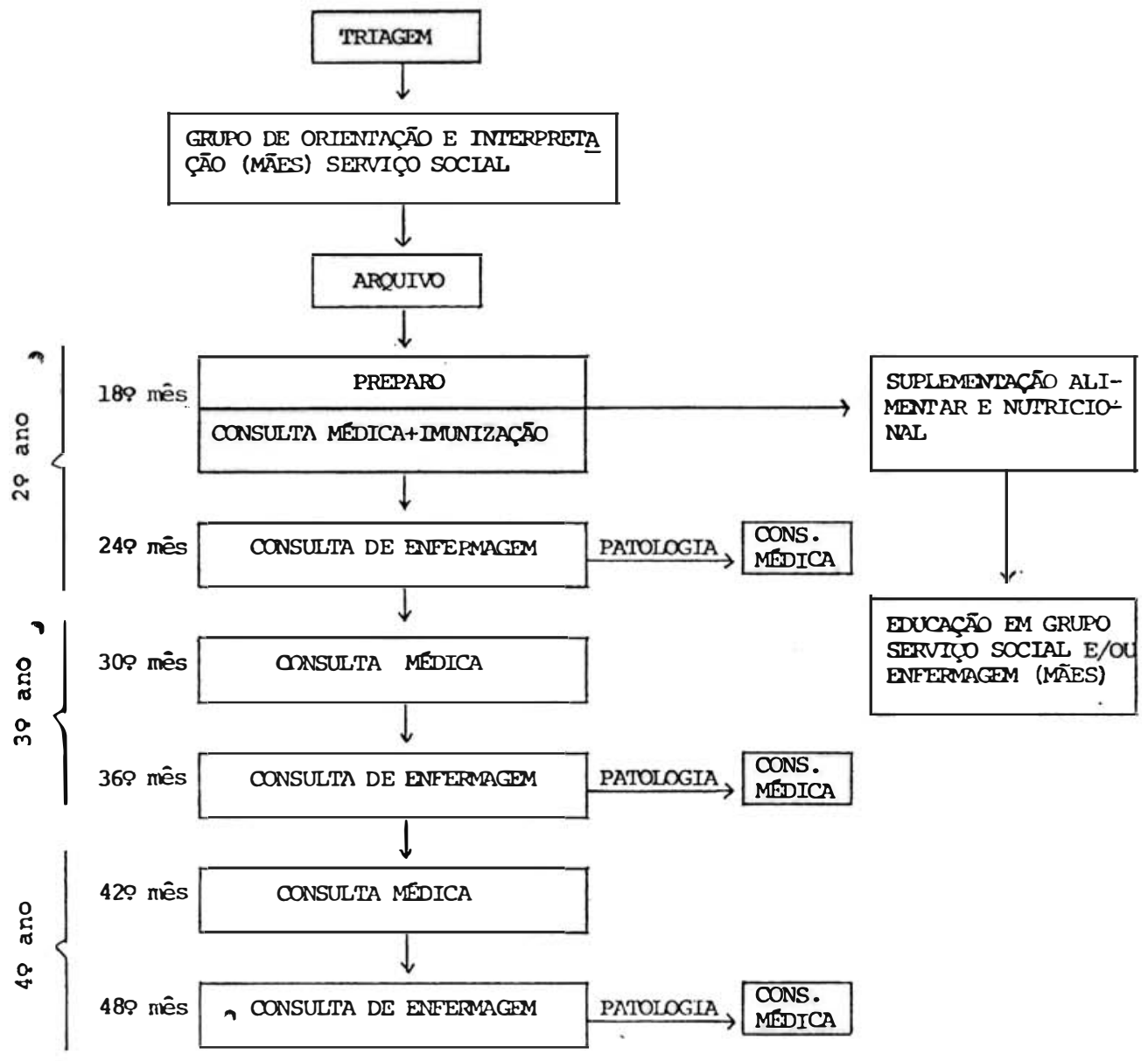


HIRATA, M.C. - Participaçāo da enfermagem no programa materno-infantil da Secretaria de Saúde do Pará. Rev. Bras. Enf.; DF, 32 : 61-74, 1979.

\section{2 - Criança de 1 a 4 anos}

A criança de 1 a 4 anos recebe o seguinte controle:

CALENDÁRIO INFANTIL (de 1 a 4 anos)

\begin{tabular}{|c|c|c|c|c|c|c|}
\hline \multirow{2}{*}{ Anos } & & \multicolumn{5}{|c|}{ C O N T R O L E } \\
\cline { 2 - 7 } & Mêses & Médico & Enferm & Imuniz & Laborat & Odont. \\
\hline \multirow{3}{*}{39} & 189 & $\mathrm{X}$ & & $\mathrm{X}$ & & \\
\cline { 2 - 8 } 39 & 249 & & $\mathrm{X}$ & & & \\
\hline & 309 & $\mathrm{X}$ & & & & \\
\hline \multirow{3}{*}{49} & 369 & & $\mathrm{X}$ & & & \\
\cline { 2 - 8 } & 429 & $\mathrm{X}$ & & & & \\
\hline
\end{tabular}

5.3 - Criança de 5 a 14 anos

A assistência à criança de 5 a 14 anos é realizada nas escolas do Estado com a participação da Secretaria de Educação, através do PRIESE (Programa Integrado de Educação e Saúde do Escolar), oferecendo a este grupo açōes de saúde como: prevenção da cárie dentária, exame físico, imunização, educação para a saúde, e recuperação, através de uma equipe multiprofissional.

\section{CONCLUSAO}

A avaliação contínua da eficiência do Programa tem permitido identificar problemas e reajustar a programação das atividades em função das discrepâncias observadas.

Quanto à eficácia do Programa Materno-Infantil, considerando-se o conjunto de variáveis que interferem nas condições de saúde do grupo em ques - tāo, dificilmente poderia ser objeto de medida. Entretanto, observando-se alguns coeficientes relativos aos três últimos anos, conclui-se que as atividades desenvolvidas com a participação ativa da enfermagem, contribuem para a melhoria do nível de saúde da população materno-infantil, sendo difícil qualificá-las de maneira específica devido à interdependência dos fatores condicionantes.

\section{BIBLIOGRAFIA}

BRASIL - Secretaria Nacional de Saúde. Coordenação de Proteçāo MaternoInfantil. Programa de Saúde MaternoInfantil. 1975.

LEAVELL, H. R. \& CLARK, G. R. - Medicina Preventiva. 3 ed. New York, Mc Grawhill, 1965.

NASCIMENTO, RODOLFO DOS SANTOS \& RAMOS, REINALDO. Evoluçĩo Histórica da Saúde Pública.
PARA - Secretaria de Estado de Saúde Pública. Coordenaçāo do Programa Materno-Infantil. Programa de Saúde Materno-Infantil. 1976/78.

PARA - Secretaria de Estado de Saúde Pública. Manual de Indicadores de Saúde, niveis e estruturas de saúde $\mathrm{e}$ saneamento, periodo 1975/77. Belém. 1978. 